\title{
Skew quantum Murnaghan-Nakayama rule
}

\author{
Matjaž Konvalinka ${ }^{1}$ \\ ${ }^{1}$ Department of Mathematics, University of Ljubljana, and Institute for Mathematics, Physics and Mechanics, Ljubl- \\ jana, Slovenia
}

\begin{abstract}
In this extended abstract, we extend recent results of Assaf and McNamara, the skew Pieri rule and the skew Murnaghan-Nakayama rule, to a more general identity, which gives an elegant expansion of the product of a skew Schur function with a quantum power sum function in terms of skew Schur functions. We give two proofs, one completely bijective in the spirit of Assaf-McNamara's original proof, and one via Lam-Lauve-Sotille's skew Littlewood-Richardson rule.

Résumé. Dans cet article nous élargissons le cadre de résultats récents de Assaf et McNamara, la règle dissymétrique de Pieri et la règle dissymétrique de Murnaghan-Nakayama, pour obtenir une identité plus générale donnant un développement élégant du produit de la fonction de Schur dissymétrique par une somme de puissances quantiques, en termes de fonctions de Schur dissymétriques. Nous donnons deux démonstrations, la première suivant l'approche de Assaf-McNamara et la deuxième par le biais de la règle dissymétrique de Littlewood-Richardson obtenue par Lam-Lauve-Sotille.
\end{abstract}

Keywords: Murnaghan-Nakayama rule, Pieri rule, skew tableaux, Schur functions, $q$-analogue

\section{Introduction}

Let us start with some basic definitions. A partition $\lambda$ of $n$ is an integer sequence $\left(\lambda_{1}, \lambda_{2}, \ldots, \lambda_{\ell}\right)$ satisfying $\lambda_{1} \geq \lambda_{2} \geq \ldots \geq \lambda_{\ell}>0$ and $\lambda_{1}+\lambda_{2}+\ldots+\lambda_{\ell}=n$; we use the notation $\lambda \vdash n, \ell=\ell(\lambda)$ (length of $\lambda$ ), $n=|\lambda|$ (size of $\lambda$ ), $\lambda_{i}=0$ if $i>\ell(\lambda)$. We sometimes write $\left(\lambda_{1}^{k_{1}}, \lambda_{2}^{k_{2}}, \ldots\right.$ ) if $\lambda_{1}$ is repeated $k_{1}$ times, $\lambda_{2}<\lambda_{1}$ is repeated $k_{2}$ times etc. The conjugate partition of $\lambda$, denoted $\lambda^{c}$, is the partition $\mu=\left(\mu_{1}, \mu_{2}, \ldots, \mu_{\lambda_{1}}\right)$ defined by $\mu_{i}=\max \left\{j: \lambda_{j} \geq i\right\}$. The Young diagram $[\lambda]$ of a partition $\lambda$ is the set $\left\{(i, j): 1 \leq i \leq \ell(\lambda), 1 \leq j \leq \lambda_{i}\right\}$. For partitions $\lambda, \mu$ we say that $\mu \subseteq \lambda$ if $\mu_{i} \leq \lambda_{i}$ for all $i$. If $\mu \subseteq \lambda$, the skew Young diagram $[\lambda / \mu]$ of $\lambda / \mu$ is the set $\left\{(i, j): 1 \leq i \leq \ell(\lambda), \mu_{i}<j \leq \lambda_{i}\right\}$. We denote $|\lambda|-|\mu|$ by $|\lambda / \mu|$. The elements of $[\lambda / \mu]$ are called cells. We treat $\lambda$ and $\lambda / \emptyset$ as identical.

We say that $\lambda / \mu$ is a horizontal strip (respectively vertical strip) if $[\lambda / \mu]$ contains no $2 \times 1$ (respectively $1 \times 2$ ) block, equivalently, if $\lambda_{i}^{c} \leq \mu_{i}^{c}+1$ (respectively $\lambda_{i} \leq \mu_{i}+1$ ) for all $i$. We say that $\lambda / \mu$ is a ribbon if $[\lambda / \mu]$ is connected and if it contains no $2 \times 2$ block, and that $\lambda / \mu$ is a broken ribbon if $[\lambda / \mu]$ contains no $2 \times 2$ block, equivalently, if $\lambda_{i} \leq \mu_{i-1}+1$ for $i \geq 2$. The Young diagram of a broken ribbon is a disjoint union of $\operatorname{rib}(\lambda / \mu)$ number of ribbons. The height $\mathrm{ht}(\lambda / \mu)$ (respectively width $\mathrm{wt}(\lambda / \mu)$ ) of a ribbon is the number of non-empty rows (respectively columns) of $[\lambda / \mu]$, minus 1 . The height (respectively width) of a broken ribbon is the sum of heights (respectively widths) of the components. Clearly, $\lambda / \mu$ is a horizontal (respectively vertical) strip if and only if it is a broken ribbon of height (respectively width) 0 . 
A map $T:[\lambda / \mu] \rightarrow \mathbb{N}$ is called a semistandard Young tableau of shape $\lambda / \mu$ if $T_{i, j_{1}} \leq T_{i, j_{2}}$ for $j_{1}<j_{2}$, and $T_{i_{1}, j}<T_{i_{2}, j}$ for $i_{1}<i_{2}$. If $T$ is bijective and maps to $\{1, \ldots,|\lambda|-|\mu|\}$, we say that it is a standard Young tableau. If $T$ is a semistandard Young tableau, we denote by $t_{i}(T)$ the number of cells that map to $i$. Define the skew Schur function $s_{\lambda / \mu}=\sum_{T} x_{1}^{t_{1}(T)} x_{2}^{t_{2}(T)} \ldots$, where the sum is over all semistandard Young tableaux of shape $\lambda / \mu$. A skew Schur function is a formal power series in $x_{1}, x_{2}, \ldots$, and it is easy to see that it is a symmetric function. Moreover, the set of Schur functions $\left\{s_{\lambda}: \lambda\right.$ partition $\}$ is a basis of the space of symmetric functions. For more details, and for some of the amazing properties of Schur functions, see [7, §7].

There are several other bases of the space of symmetric functions. For the purposes of this paper, the most important one is the power sum basis $\left\{p_{\lambda}: \lambda\right.$ partition $\}$, defined by $p_{r}=x_{1}^{r}+x_{2}^{r}+\ldots$, $p_{\lambda}=p_{\lambda_{1}} p_{\lambda_{2}} \cdots p_{\lambda_{\ell}}$. Let us also mention the monomial basis $\left\{m_{\lambda}: \lambda\right.$ partition $\}$, defined by $m_{\lambda}=$ $\sum x_{\pi(1)}^{\lambda_{1}} \cdots x_{\pi(\ell)}^{\lambda_{\ell}}$, where the sum is over all injective maps $\pi:\{1, \ldots, \ell\} \rightarrow \mathbb{N}$.

The product of Schur functions can be uniquely expressed as a linear combination of Schur functions: $s_{\lambda} s_{\mu}=\sum c_{\lambda, \mu}^{\nu} s_{\nu}$. The coefficients $c_{\lambda, \mu}^{\nu}$ are called Littlewood-Richardson coefficients and can be computed using the celebrated Littlewood-Richardson rule, see [7, Appendix A1.3]. This rule is quite complicated, but it is very simple if $\mu$ has only one row or column. Namely, we have the Pieri rule $-P R$ :

$$
s_{\lambda} \cdot s_{r}=\sum s_{\lambda^{+}}
$$

where the sum on the right is over all $\lambda^{+}$such that $\lambda^{+} / \lambda$ is a horizontal strip of size $r$. Similarly, the conjugate Pieri rule $-C P R$ says that

$$
s_{\lambda} \cdot s_{1^{r}}=\sum s_{\lambda^{+}},
$$

where the sum on the right is over all $\lambda^{+}$such that $\lambda^{+} / \lambda$ is a vertical strip of size $r$. See [7, §7.15].

The Murnaghan-Nakayama rule - MNR gives the product of a Schur function with a power sum symmetric function:

$$
s_{\lambda} \cdot p_{r}=\sum(-1)^{\operatorname{ht}\left(\lambda^{+} / \lambda\right)} s_{\lambda^{+}},
$$

where the sum on the right is over all $\lambda^{+}$such that $\lambda^{+} / \lambda$ is a ribbon of size $r$. See [7, §7.17].

In [1] and [2], Assaf and McNamara found a beautiful extension of both PR and MNR.

Theorem 1 (Skew Pieri rule - SPR) For partitions $\lambda, \mu, \mu \subseteq \lambda$, we have

$$
s_{\lambda / \mu} \cdot s_{r}=\sum_{j}(-1)^{j} \sum s_{\lambda^{+} / \mu^{-}},
$$

where the inner sum on the right is over all $\lambda^{+}, \mu^{-}$such that $\lambda^{+} / \lambda$ is a horizontal strip of size $r-j$, and $\mu / \mu^{-}$is a vertical strip of size $j$.

Corollary 2 (Conjugate skew Pieri rule - CSPR) For partitions $\lambda, \mu, \mu \subseteq \lambda$, we have

$$
s_{\lambda / \mu} \cdot s_{1^{r}}=\sum_{j}(-1)^{j} \sum s_{\lambda^{+} / \mu^{-}},
$$

where the inner sum on the right is over all $\lambda^{+}, \mu^{-}$such that $\lambda^{+} / \lambda$ is a vertical strip of size $r-j$, and $\mu / \mu^{-}$is a horizontal strip of size $j$ 
CSPR can be proved from SPR via the involution $\omega$ on the algebra of symmetric functions which maps $s_{\lambda / \mu}$ to $s_{\lambda^{c} / \mu^{c}}$ and preserves the product. See [7, $\$ 7.6$ and $\S 7.14$ ] for details.

Theorem 3 (Skew Murnaghan-Nakayama Rule - SMNR) For partitions $\lambda, \mu, \mu \subseteq \lambda$, we have

$$
s_{\lambda / \mu} \cdot p_{r}=\sum(-1)^{\mathrm{ht}\left(\lambda^{+} / \lambda\right)} s_{\lambda^{+} / \mu}-\sum(-1)^{\mathrm{ht}\left(\mu / \mu^{-}\right)} s_{\lambda / \mu^{-}}
$$

where the first (respectively second) sum on the right is over all $\lambda^{+}$(respectively $\mu^{-}$) such that $\lambda^{+} / \lambda$ (respectively $\mu / \mu^{-}$) is a ribbon of size $r$.

Note that while the Pieri rule and the Murnaghan-Nakayama rule give the expansion in terms of a basis, their skew versions give only one possible (but obviously special) expansion in terms of skew Schur functions, which are not a basis of the space of symmetric functions. Assaf and McNamara provide an elegant bijective proof of their skew Pieri rule (but not of the skew Murnaghan-Nakayama rule).

Define quantum power sum symmetric functions by

$$
\widetilde{p}_{r}=\sum_{\tau \vdash r}(-1)^{\ell(\tau)-1}(q-1)^{\ell(\tau)-1} m_{\tau}, \quad \widetilde{p}_{\lambda}=\widetilde{p}_{\lambda_{1}} \widetilde{p}_{\lambda_{2}} \cdots
$$

The functions $\widetilde{p}_{\lambda}$ have connections with representation theory (more precisely, characters of the Hecke algebra of type A; see for example [3, Theorem 6.5.3]). We have $\left.\widetilde{p}_{r}\right|_{q=1}=m_{r}=p_{r},\left.\widetilde{p}_{r}\right|_{q=0}=$ $\sum_{\tau \vdash r} m_{\tau}=s_{r}, \lim _{q \rightarrow \infty} \frac{\widetilde{p}_{r}}{q^{r-1}}=(-1)^{r-1} m_{1^{r}}=(-1)^{r-1} s_{1^{r}}$.

There exists a very natural generalization of the Murnaghan-Nakayama rule, the quantum MurnaghanNakayama rule - QMNR:

$$
s_{\lambda} \cdot \widetilde{p}_{r}=(-1)^{r+1} \sum(-1)^{\mathrm{wt}\left(\lambda^{+} / \lambda\right)} q^{\mathrm{ht}\left(\lambda^{+} / \lambda\right)}(q-1)^{\mathrm{rib}\left(\lambda^{+} / \lambda\right)-1} s_{\lambda^{+}}
$$

where the internal sum on the right is over $\lambda^{+}$such that $\lambda^{+} / \lambda$ is a broken ribbon of size $r$. See for example [3, Theorem 6.5.2] for a slightly different version and a (complicated) bijective proof via the classical definition of Schur functions.

The following is our main result, the skew quantum Murnaghan-Nakayama rule.

Theorem 4 (SQMNR) For partitions $\lambda, \mu, \mu \subseteq \lambda$, and $r \geq 0$, we have

$s_{\lambda / \mu} \cdot \widetilde{p}_{r}=\sum_{j=0}^{r}(-1)^{r+1-j} \sum(-1)^{\operatorname{wt}\left(\lambda^{+} / \lambda\right)+\operatorname{ht}\left(\mu / \mu^{-}\right)} q^{\operatorname{ht}\left(\lambda^{+} / \lambda\right)+\operatorname{wt}\left(\mu / \mu^{-}\right)}(q-1)^{\operatorname{rib}\left(\lambda^{+} / \lambda\right)+\operatorname{rib}\left(\mu / \mu^{-}\right)-1} s_{\lambda^{+} / \mu^{-}}$,

where the internal sum on the right is over $\lambda^{+}, \mu^{-}$such that $\lambda^{+} / \lambda$ is a broken ribbon of size $r-j$, and $\mu / \mu^{-}$is a broken ribbon of size $j$.

There is an equivalent version of the statement that will be slightly more useful for our purposes.

Theorem 5 (SQMNR') For partitions $\lambda, \mu, \mu \subseteq \lambda$, and $r \geq 0$, we have

$$
s_{\lambda / \mu} \cdot \widetilde{p}_{r}=\sum(-1)^{\left|\mu / \mu^{-}\right|}(-q)^{\operatorname{ht}\left(\lambda^{+} / \lambda\right)+\operatorname{wt}\left(\mu / \mu^{-}\right)}(1-q)^{\operatorname{rib}\left(\lambda^{+} / \lambda\right)+\operatorname{rib}\left(\mu / \mu^{-}\right)-1} s_{\lambda^{+} / \mu^{-}},
$$

where the sum is over $\lambda^{+}, \mu^{-}$such that $\lambda^{+} / \lambda$ and $\mu / \mu^{-}$are broken ribbons with $\left|\lambda^{+} / \lambda\right|+\left|\mu / \mu^{-}\right|=r$. 
The main theorem is a generalization of several statements. The following is a sample:

- $q=0$ : a term on the right-hand side of SQMNR' is non-zero if and only if $\operatorname{ht}\left(\lambda^{+} / \lambda\right)+\mathrm{wt}\left(\mu / \mu^{-}\right)=$ 0 . In this case, $\lambda^{+} / \lambda$ has height 0 (and is a horizontal strip) and $\mu / \mu^{-}$has width 0 (and is a vertical strip). As noted above, $\left.\widetilde{p}_{r}\right|_{q=0}=s_{r}$. SQMNR' specializes to SPR due to Assaf-McNamara [1].

- $q=1$ : a term on the right of SQMNR' is non-zero if and only if $\operatorname{rib}\left(\lambda^{+} / \lambda\right)+\operatorname{rib}\left(\mu / \mu^{-}\right)-1=0$. In this case, one of $\lambda^{+} / \lambda$ and $\mu / \mu^{-}$is empty, and the other one is a ribbon. We know that $\left.\widetilde{p}_{r}\right|_{q=1}=p_{r}$. It is easy to see that SQMNR' becomes SMNR due to Assaf-McNamara [2].

- $q \rightarrow \infty$ : divide SQMNR by $q^{r-1}$ and let $q \rightarrow \infty$. It is easy to prove that this simplifies to CSPR.

- $\mu=\emptyset$ : SQMNR is QMNR; we get the classical PR for $q=0$, the classical MNR for $q=1$, and the classical CPR if we let $q \rightarrow \infty$.

- $\lambda=\mu=\emptyset$ : this gives the expansion of quantum power sum functions in the basis of Schur functions, $\widetilde{p}_{r}=\sum_{k=1}^{r}(-q)^{r-k} s_{k, 1^{r-k}}$. We will verify this independently in Lemma 8 .

This extended abstract is structured as follows. In Section 2, we describe the sign-reversing involution of Assaf and McNamara that was used to prove their skew Pieri rule. Furthermore, we show a variant of this involution that proves the conjugate skew Pieri rule. Note that this involution is actually simpler than the one in [1] (but, of course, does not provide a bijective proof of the skew Pieri rule itself). In Section 3. we present an extension of these involutions that proves the skew quantum Murnaghan-Nakayama rule. There is quite some work involved to interpret the right-hand side of SQMNR in an appropriate way, but once this is done the involution is just a natural combination of the two involutions in Section 2 In Section 4. we sketch another proof of SQMNR, via the skew Littlewood-Richardson rule of Lam-Lauve-Sotille [5]; since this result (at the moment) only has an algebraic proof, this proof of SQMNR is not completely combinatorial. We finish with some concluding remarks in Section 5 A reader interested in a brief history of the subject and all the necessary references should consult [1]. The full version of this paper is [4].

\section{Proofs of the skew Pieri rule and its dual}

One of the most important algorithms on semistandard Young tableaux is the Robinson-Schensted row insertion. Given a semistandard Young tableau $T$ of shape $\lambda$ and an integer $k$, we can insert $k$ into $T$ as follows. Define $k_{1}=k$. Find the smallest $j$ so that $T_{1 j}>k_{1}$, replace $T_{1 j}$ by $k_{1}$, and define $k_{2}$ to be the previous value of $T_{1 j}$. Then find the smallest $j$ so that $T_{2 j}>k_{2}$, replace $T_{2 j}$ by $k_{2}$, and define $k_{3}$ to be the previous value of $T_{2 j}$. Continue until, for some $i^{\prime}$, all elements of row $i^{\prime}$ are $\leq k_{i^{\prime}}$. Then define $T_{i^{\prime}, \lambda_{i}^{\prime}+1}=k_{i^{\prime}}$, and finish the algorithm. The result is again a semistandard Young tableau. We say that the insertion of $k$ into $T$ exits in row $i^{\prime}$. See [7, §7.11] for details.

Now assume we have a semistandard Young tableau $T$ of some skew shape $\lambda / \mu$. We can insert $k$ into $T$ for some integer $k$ in almost exactly the same way. Define $k_{1}=k$. Find the smallest $j, \mu_{1}<j \leq \lambda_{1}$, so that $T_{1 j}>k_{1}$, replace $T_{1 j}$ by $k_{1}$, and define $k_{2}$ to be the previous value of $T_{1 j}$. Then find the smallest $j$, $\mu_{2}<j \leq \lambda_{2}$, so that $T_{2 j}>k_{2}$, replace $T_{2 j}$ by $k_{2}$, and define $k_{3}$ to be the previous value of $T_{2 j}$. Continue until, for some $i^{\prime}$, all elements of row $i^{\prime}$ are $\leq k_{i^{\prime}}$. Then define $T_{i^{\prime}, \lambda_{i^{\prime}}+1}=k_{i^{\prime}}$, and finish the algorithm. The result is again a semistandard Young tableau. We say that the insertion of $k$ into $T$ exits in row $i^{\prime}$. 
There is another natural kind of insertion. Take $i_{0}$ so that $\mu_{i_{0}}<\lambda_{i_{0}}$ and either $i_{0}=1$ or $\mu_{i_{0}-1}>\mu_{i_{0}}$, and take $k_{i_{0}+1}=T_{i_{0}, \mu_{i_{0}}+1}$. We can insert from row $i_{0}$ in $T$ as follows. Erase the entry $T_{i_{0}, \mu_{i_{0}}+1}$. Find the smallest $j, \mu_{i_{0}+1}<j \leq \lambda_{i_{0}+1}$, so that $T_{i_{0}+1, j}>k_{i_{0}+1}$, replace $T_{i_{0}+1, j}$ by $k_{i_{0}+1}$, and define $k_{i_{0}+2}$ to be the previous value of $T_{i_{0}+1, j}$. Then find the smallest $j, \mu_{i_{0}+2}<j \leq \lambda_{i_{0}+2}$, so that $T_{i_{0}+2, j}>k_{i_{0}+2}$, replace $T_{i_{0}+2, j}$ by $k_{i_{0}+2}$, and define $k_{i_{0}+3}$ to be the previous value of $T_{i_{0}+2, j}$. Continue until, for some $i^{\prime}$, all elements of row $i^{\prime}$ are $\leq k_{i^{\prime}}$. Then define $T_{i^{\prime}, \lambda_{i^{\prime}}+1}=k_{i^{\prime}}$, and finish the algorithm. The result is again a semistandard Young tableau. We say that the insertion from row $i_{0}$ in $T$ exits in row $i^{\prime}$.
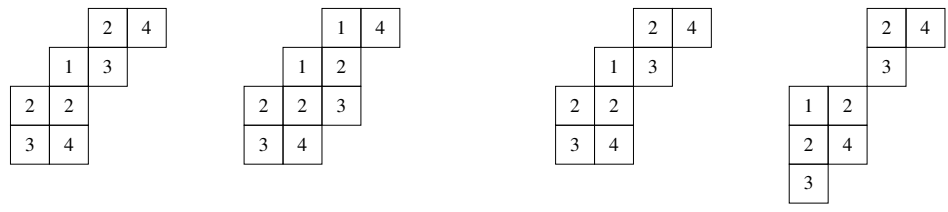

Fig. 1: Insertion of 1 into a tableau, and insertion from row 2 in a tableau.

Insertion has an inverse operation, reverse insertion. Say we are given a semistandard Young tableau $T$ of shape $\lambda / \mu$. Take $i^{\prime}$ so that $\lambda_{i^{\prime}+1}<\lambda_{i^{\prime}}$. We reverse insert from row $i^{\prime}$ in $T$ as follows. Define $k_{i^{\prime}-1}=T_{i^{\prime}, \lambda_{i^{\prime}}}$. Erase the entry $T_{i^{\prime}, \lambda_{i^{\prime}}}$. Find the largest $j, \mu_{i^{\prime}-1}<j \leq \lambda_{i^{\prime}-1}$, so that $T_{i^{\prime}-1, j}<k_{i^{\prime}-1}$, replace $T_{i^{\prime}-1, j}$ by $k_{i^{\prime}-1}$, and define $k_{i^{\prime}-2}$ to be the previous value of $T_{i^{\prime}-1, j}$. Then find the largest $j$, $\mu_{i^{\prime}-2}<j \leq \lambda_{i^{\prime}-2}$, so that $T_{i^{\prime}-2, j}<k_{i^{\prime}-2}$, replace $T_{i^{\prime}-2, j}$ by $k_{i^{\prime}-2}$, and define $k_{i^{\prime}-3}$ to be the previous value of $T_{i^{\prime}-2, j}$. Continue until we have $k_{i_{0}}$, where either $i_{0}=0$ or all elements of row $i_{0}$ are $\geq k_{i_{0}}$. If $i_{0}=0$, the result is a pair $(S, k)$, where $S$ is a semistandard Young tableau and $k=k_{0}$. We call $k$ the exiting integer. If $i_{0} \geq 1$ and all elements of row $i_{0}$ are $\geq k_{i_{0}}$, define $T_{\mu_{i_{0}}}=k_{i_{0}}$. The result is a semistandard Young tableau $S$. We say that the reverse insertion from row $i^{\prime}$ in $T$ exits in row $i_{0}$.
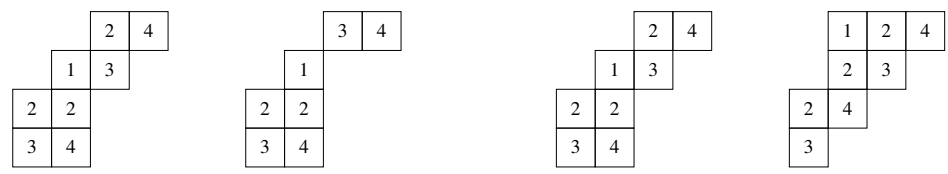

Fig. 2: Reverse insertion from rows 2 (which exits in row 0 with exiting integer 2) and 4 (which exits in row 1).

In [1], the operations of insertion and reverse insertion are proved to be inverses of one another in the following sense. If the insertion of an integer $k$ into a semistandard Young tableau $T$ exits in row $i^{\prime}$ and the resulting tableau is $S$, then the reverse insertion from row $i^{\prime}$ in $S$ exits in row 0 and the result is $(T, k)$. If the insertion from row $i_{0}$ into $T$ exits in row $i^{\prime}$ and the resulting tableau is $S$, then the reverse insertion from row $i^{\prime}$ in $S$ exits in row $i_{0}$ and the result is $T$. Similarly, if the reverse insertion from row $i^{\prime}$ in $T$ exits in row 0 and the result is $(S, k)$, then the insertion of $k$ into $S$ exits in row $i^{\prime}$ and the result is $T$. And if the reverse insertion from row $i^{\prime}$ in $T$ exits in row $i_{0} \geq 1$ and the result is $S$, then the insertion from row $i_{0}$ into $S$ exits in row $i^{\prime}$ and the result is $T$.

The involution by Assaf and McNamara which proves the skew Pieri rule works as follows. Say we are given a skew shape $\lambda / \mu$ and a semistandard Young tableau $T$ of shape $\lambda^{+} / \mu^{-}$, where $\lambda^{+} / \lambda$ is a horizontal strip and $\mu / \mu^{-}$is a vertical strip. Let $v$ be the empty word. Let $i=\infty$ if $\mu=\mu^{-}$, and let $i$ 
be the top row of $\mu / \mu^{-}$otherwise. While $\lambda^{+} \neq \lambda$ and the reverse insertion from row $i^{\prime}$, the top row of $\lambda^{+} / \lambda$, in $T$ exits in row 0 and results in $(S, k)$, attach $k$ to the beginning of $v$, let $T=S$, and let $\lambda^{+} / \mu^{-}$ be the shape of the new $T$ (note that $\lambda_{i^{\prime}}^{+}$is decreased by 1 and $\mu^{-}$remains the same). If the while loop stops when $\lambda^{+} \neq \lambda$ and the reverse insertion from row $i^{\prime}$ in $T$ exits in row $i_{0}, 0<i_{0}<i$, and results in $S$, let $T=S$. If the while loop stops when $\lambda^{+}=\lambda, \mu \neq \mu^{-}$, or when $\lambda^{+} \neq \lambda$ and the reverse insertion from row $i^{\prime}$ in $T$ exits in row $i_{0}, i_{0} \geq i$, insert from row $i$ in $T$ and call the resulting tableau $T$. Finish the algorithm by inserting the entries of $v$ from left to right into $T$. The final result is a semistandard Young tableau of some shape $\lambda^{++} / \mu^{--}$, we denote it $\Phi_{\lambda, \mu, \lambda^{+}, \mu^{-}}(T)$.

It turns out that $\Phi$ is an involution, and $T$ is a fixed point if and only if $\mu=\mu^{-}$and the while loop stops when $\lambda^{+}=\lambda$. Such fixed points are in one-to-one correpondence with pairs $(S, v)$, where $S$ is a semistandard Young tableau of shape $\lambda / \mu$ and $v$ is a weakly increasing word. Indeed, if we stop the algorithm after the while loop, we have exactly such a pair, and given a pair $(S, v)$, we can insert the entries of $v$ from left to right into $S$ to get the corresponding $T$. Furthermore, if $T$ is not a fixed point, then $\left|\mu^{--}\right|=\left|\mu^{-}\right| \pm 1$. It is easy to see that this shows the skew Pieri rule. See [1] for details and a precise proof, and [1], [2] or [4] for examples.

As mentioned in the introduction, the conjugate skew Pieri rule follows from SPR by applying the involution $\omega$ on the algebra of symmetric functions. There is, however, an involution in the spirit of Assaf-McNamara that proves CSPR.

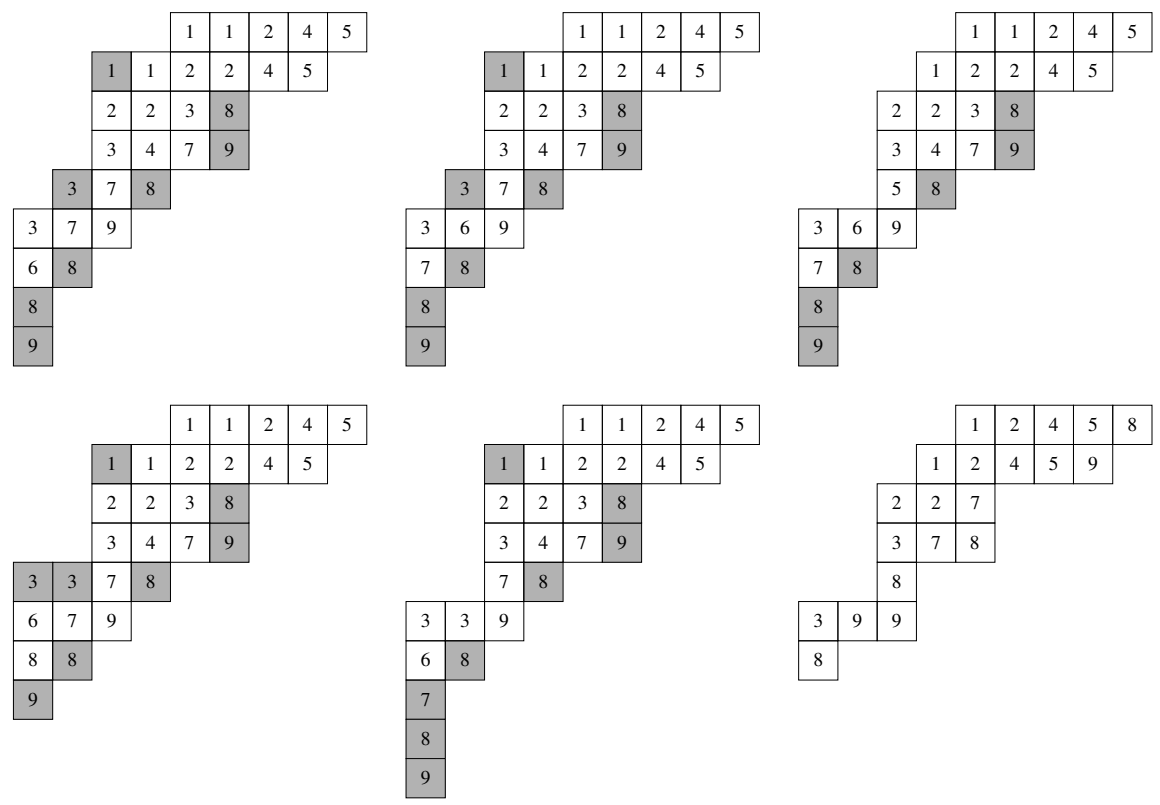

Fig. 3: Tableaux $T_{1}, T_{2}, T_{3}$ are on top; $\Psi\left(T_{1}\right)$ and $\Psi\left(T_{2}\right)$ are the bottom left and bottom middle tableaux, while $T_{3}$ is a fixed point of $\Psi$. The bottom right figure shows the tableau we get if we repeatedly reverse insert from the bottom row of $T_{3}$; the exiting integers are $1,2,3,4,5,6$. 
Fix $\lambda, \mu, r$. A term on the right-hand side is represented by a semistandard Young tableau of shape $\lambda^{+} / \mu^{-}$, where $\lambda^{+} / \lambda$ is a vertical strip, $\mu / \mu^{-}$is a horizontal strip, and $\left|\lambda^{+} / \lambda\right|+\left|\mu / \mu^{-}\right|=r$. Such a tableau $T$ is weighted by $(-1)^{\left|\mu / \mu^{-}\right|}$. Let $i$ denote the bottom row of $\mu / \mu^{-}$(unless $\mu=\mu^{-}$, in which case take $i=0$ ). Now reverse insert from row $i^{\prime}$, the bottom row of $\lambda^{+} / \lambda$, in $T$ (unless $\lambda^{+}=\lambda$ ). If the reverse insertion exits the diagram in row $\geq i$ (except in the case when $\mu=\mu^{-}$and the reverse insertion exits in row 0), call this new diagram $\Psi(T)=\Psi_{\lambda, \mu, \lambda^{+}, \mu^{-}}(T)$. See Figure 3, left. If this reverse insertion exits the diagram in row $<i$, or if $\lambda^{+}=\lambda$, insert from row $i$ in $T$ and call the result $\Psi(T)=\Psi_{\lambda, \mu, \lambda^{+}, \mu^{-}}(T)$. See Figure 3 middle. When $\mu=\mu^{-}$and the reverse insertion exits in row 0 , take $\Psi(T)=\Psi_{\lambda, \mu, \lambda^{+}, \mu^{-}}(T)=T$. See Figure 3 right.

Proposition 6 The map $\Psi_{\lambda, \mu, \lambda^{+}, \mu^{-}}$is an involution that is sign-reversing except on fixed points. Furthermore, the fixed points are in a bijective correspondence with elements on the left-hand side of CSPR.

\section{A bijective proof of the main theorem}

The first step of our proof is to interpret the right-hand side of SQMNR' as a weighted sum over some combinatorial objects. The appropriate objects turn out to be semistandard Young tableaux with some cells colored gray. To motivate these colorings, observe the following. If we "glue" together a vertical strip and a horizontal strip in such a way that the result is a skew diagram, then this skew diagram cannot have any $2 \times 2$ squares. In other words, it is a broken ribbon. This also holds the other way around: if we are given a broken ribbon, we can break it up into a vertical strip and a horizontal strip.

Let us multiply both sides of SQMNR' by $1-q$ and call this statement SQMNR":

$s_{\lambda / \mu} \cdot\left(\sum_{\tau \vdash r}(1-q)^{\ell(\tau)} m_{\tau}\right)=\sum_{\lambda^{+}, \mu^{-}}(-1)^{\left|\mu / \mu^{-}\right|}(-q)^{\operatorname{ht}\left(\lambda^{+} / \lambda\right)+\operatorname{wt}\left(\mu / \mu^{-}\right)}(1-q)^{\operatorname{rib}\left(\lambda^{+} / \lambda\right)+\operatorname{rib}\left(\mu / \mu^{-}\right)} s_{\lambda^{+} / \mu^{-}}$.

We have fixed $\lambda, \mu, r$. Say that we are given $\lambda^{+}, \mu^{-}$such that $\lambda^{+} / \lambda$ and $\mu / \mu^{-}$are broken ribbons with $\left|\lambda^{+} / \lambda\right|+\left|\mu / \mu^{-}\right|=r$, and a semistandard Young tableau $T$ of shape $\lambda^{+} / \mu^{-}$. Our first goal is to break up each of the broken ribbons $\lambda^{+} / \lambda$ and $\mu / \mu^{-}$into a vertical strip and a horizontal strip. More precisely, we wish to choose partitions $\lambda^{\prime}, \mu^{\prime}$ such that $\lambda^{\prime} / \lambda$ and $\mu^{\prime} / \mu^{-}$are horizontal strips, and $\lambda^{+} / \lambda^{\prime}$ and $\mu / \mu^{\prime}$ are vertical strips. We weight such a selection with

$$
(-1)^{\left|\mu / \mu^{-}\right|}(-q)^{\left|\lambda^{+} / \lambda^{\prime}\right|+\left|\mu^{\prime} / \mu^{-}\right|} .
$$

We color the cells of $\lambda^{+} / \lambda^{\prime}$ and $\mu^{\prime} / \mu^{-}$gray and leave the other cells white. So our requirements are saying that the gray cells of $\lambda^{+} / \lambda$ and the white cells of $\mu / \mu^{-}$form a vertical strip, and the white cells of $\lambda^{+} / \lambda$ and the gray cells of $\mu / \mu^{-}$form a horizontal strip; also, the white cells form a diagram of some shape $\lambda^{\prime} / \mu^{\prime}$ for $\lambda \subseteq \lambda^{\prime} \subseteq \lambda^{+}, \mu^{-} \subseteq \mu^{\prime} \subseteq \mu$. Furthermore, the weight of such an object is $(-1)^{\left|\mu / \mu^{-}\right|}(-q)^{j}$, where $j$ is the number of gray cells.

We claim that these objects indeed enumerate the right-hand side of SQMNR'.

Lemma 7 For fixed $\lambda, \mu, \lambda^{+}, \mu^{-}$, we have

$$
\sum(-1)^{\left|\mu / \mu^{-}\right|}(-q)^{\left|\lambda^{+} / \lambda^{\prime}\right|+\left|\mu^{\prime} / \mu^{-}\right|}=(-1)^{\left|\mu / \mu^{-}\right|}(-q)^{\operatorname{ht}\left(\lambda^{+} / \lambda\right)+\operatorname{wt}\left(\mu / \mu^{-}\right)}(1-q)^{\operatorname{rib}\left(\lambda^{+} / \lambda\right)+\operatorname{rib}\left(\mu / \mu^{-}\right)},
$$



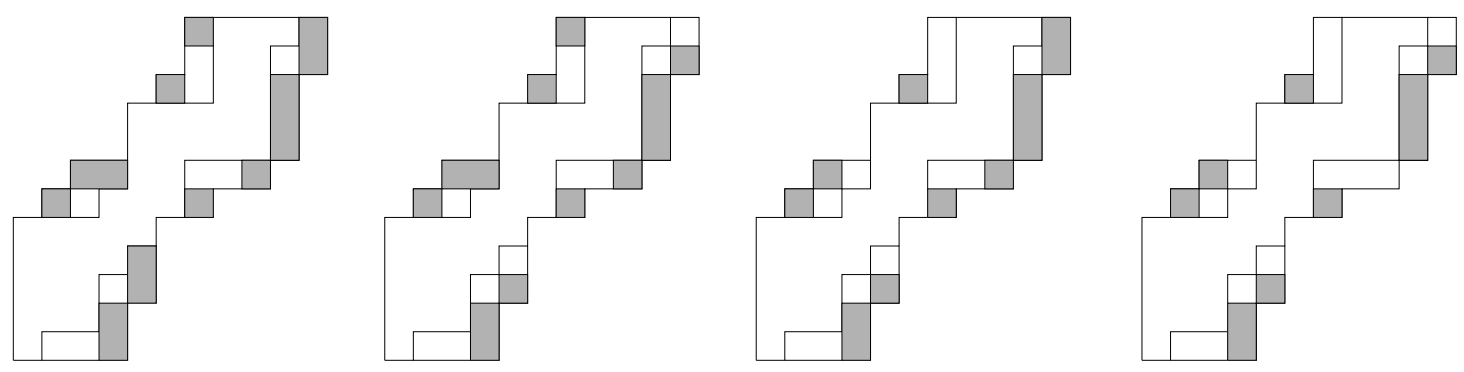

Fig. 4: Four examples with weights $q^{16}, q^{14}, q^{13}$ and $q^{11}$.

where the sum on the left runs over all $\lambda^{\prime}, \mu^{\prime}$ such that $\lambda^{\prime} / \lambda$ and $\mu^{\prime} / \mu^{-}$are horizontal strips, and $\lambda^{+} / \lambda^{\prime}$ and $\mu / \mu^{\prime}$ are vertical strips.

Proof: For each cell of $\lambda^{+} / \lambda$, we have to decide whether or not to put it in $\lambda^{\prime} / \lambda$ or in $\lambda^{+} / \lambda^{\prime}$ (i.e. whether to make it white or gray). If a cell in $\lambda^{+} / \lambda$ has a right neighbor in $\lambda^{+} / \lambda$, it cannot be in $\lambda^{+} / \lambda^{\prime}$, since its right neighbor would also have to be in $\lambda^{+} / \lambda^{\prime}$, and this would contradict the requirement that $\lambda^{+} / \lambda^{\prime}$ is a vertical strip. Similarly, if a cell in $\lambda^{+} / \lambda$ has an upper neighbor in $\lambda^{+} / \lambda$, it cannot be in $\lambda^{\prime} / \lambda$, since its upper neighbor would also have to be in $\lambda^{\prime} / \lambda$, and this would contradict the requirement that $\lambda^{\prime} / \lambda$ is a horizontal strip.

This means that the colors of all the cells in $\lambda^{+} / \lambda$ are determined, except for the top right cell of each ribbon of $\lambda^{+} / \lambda$, which can be either white or gray.

If a cell in $\mu / \mu^{-}$has a right neighbor in $\mu / \mu^{-}$, it cannot be in $\mu / \mu^{\prime}$, since its right neighbor would also have to be in $\mu / \mu^{\prime}$, and this would contradict the requirement that $\mu / \mu^{\prime}$ is a vertical strip. Similarly, if a cell in $\mu / \mu^{-}$has an upper neighbor in $\mu / \mu^{-}$, it cannot be in $\mu^{\prime} / \mu^{-}$, since its upper neighbor would also have to be in $\mu^{\prime} / \mu^{-}$, and this would contradict the requirement that $\mu^{\prime} / \mu^{-}$is a horizontal strip.

This means that the colors of all the cells in $\mu / \mu^{-}$are determined, except for the top right cell of each ribbon of $\mu / \mu^{-}$, which can be either white or gray.

In other words, we have two choices for each top right cell of each ribbon of $\left(\lambda^{+} / \lambda\right) \cup\left(\mu / \mu^{-}\right)$. We have at least $\mathrm{ht}\left(\lambda^{+} / \lambda\right)$ gray cells in $\lambda^{+} / \lambda$, and at least $\mathrm{wt}\left(\mu / \mu^{-}\right)$gray cells in $\mu / \mu^{-}$. So the weight of a term on the left-hand side is $(-1)^{\left|\mu / \mu^{-}\right|}(-q)^{\operatorname{ht}\left(\lambda^{+} / \lambda\right)+\operatorname{wt}\left(\mu / \mu^{-}\right)}(-q)^{j}$, where $j$ is the number of cells that are gray by choice, and these choices are made independently. Of course,

$$
\begin{gathered}
\sum_{j}\left(\begin{array}{c}
\operatorname{rib}\left(\lambda^{+} / \lambda\right)+\operatorname{rib}\left(\mu / \mu^{-}\right) \\
j
\end{array}\right)(-1)^{\left|\mu / \mu^{-}\right|}(-q)^{\operatorname{ht}\left(\lambda^{+} / \lambda\right)+\operatorname{wt}\left(\mu / \mu^{-}\right)}(-q)^{j}= \\
=(-1)^{\left|\mu / \mu^{-}\right|}(-q)^{\operatorname{ht}\left(\lambda^{+} / \lambda\right)+\operatorname{wt}\left(\mu / \mu^{-}\right)}(1-q)^{\operatorname{rib}\left(\lambda^{+} / \lambda\right)+\operatorname{rib}\left(\mu / \mu^{-}\right)},
\end{gathered}
$$

which finishes the proof of the lemma.

We have managed to rewrite SQMNR" as follows:

$$
s_{\lambda / \mu} \cdot\left(\sum_{\tau \vdash r}(1-q)^{\ell(\tau)} m_{\tau}\right)=\sum(-1)^{\left|\mu / \mu^{-}\right|}(-q)^{\left|\lambda^{+} / \lambda^{\prime}\right|+\left|\mu^{\prime} / \mu^{-}\right|} s_{\lambda^{+} / \mu^{-}},
$$


where the sum is over all partitions $\lambda^{+}, \lambda^{\prime}, \mu^{-}, \mu^{\prime}$ such that $\lambda^{+} / \lambda$ and $\mu / \mu^{-}$are broken ribbons with $\left|\lambda^{+} / \lambda\right|+\left|\mu / \mu^{-}\right|=r, \lambda^{\prime} / \lambda$ and $\mu^{\prime} / \mu^{-}$are horizontal strips, and $\lambda^{+} / \lambda^{\prime}$ and $\mu / \mu^{\prime}$ are vertical strips.

For fixed $\lambda, \mu, r$, a term on the right-hand side of SQMNR" therefore corresponds to a semistandard Young tableau $T$ with some cells colored white and some cells colored gray, such that the following properties are satisfied:

- the shape of $T$ is $\lambda^{+} / \mu^{-}$for some $\lambda^{+} \supseteq \lambda$ and $\mu^{-} \subseteq \mu,\left|\lambda^{+} / \lambda\right|+\left|\mu / \mu^{-}\right|=r$, and $\lambda^{+} / \lambda$ and $\mu / \mu^{-}$are broken ribbons;

- the white cells form a skew diagram $\lambda^{\prime} / \mu^{\prime}$ for some partitions $\lambda^{\prime}, \mu^{\prime}, \lambda^{\prime} \supseteq \lambda, \mu^{\prime} \subseteq \mu$;

- the white cells in $\lambda^{+} / \lambda$ form a horizontal strip, and the white cells in $\mu / \mu^{-}$form a vertical strip;

- the gray cells in $\lambda^{+} / \lambda$ form a vertical strip, and the gray cells in $\mu / \mu^{-}$a horizontal strip;

We call such an object a colored tableau of shape $\left(\lambda, \mu, \lambda^{\prime}, \mu^{\prime}, \lambda^{+}, \mu^{-}\right)$. We weight a colored tableau by

$$
(-1)^{\left|\mu / \mu^{-}\right|}(-q)^{\left|\lambda^{+} / \lambda^{\prime}\right|+\left|\mu^{\prime} / \mu^{-}\right|} \text {. }
$$

Perform the involution $\Psi$ on the gray cells of a colored tableau. More specifically, find $\Psi_{\lambda^{\prime}, \mu^{\prime}, \lambda^{+}, \mu^{-}}(T)$. Since $\lambda^{+} / \lambda^{\prime}$ is a vertical strip and $\mu^{\prime} / \mu^{-}$is a horizontal strip, the map is well defined. One gray cell is removed, and one gray cell is added in the process. The result is a colored tableau $T^{\prime}$ of shape $\left(\lambda, \mu, \lambda^{\prime}, \mu^{\prime}, \lambda^{++}, \mu^{--}\right)$for some $\lambda^{++}, \mu^{--}$; it has the same white cells as $T$, the same number of gray cells as $T$, and with the property that $\left|\mu / \mu^{--}\right|=\left|\mu / \mu^{-}\right| \pm 1$ unless $T=T^{\prime}$ is a fixed point.

This already cancels a large number of terms. The ones that remain correspond to fixed points of $\Psi_{\lambda^{\prime}, \mu^{\prime}, \lambda^{+}, \mu^{-}}$. Each such fixed point consists of a a semistandard Young tableau $S$ of shape $\lambda^{\prime} / \mu^{\prime}$, where $\lambda^{\prime} / \lambda$ is a horizontal strip and $\mu / \mu^{\prime}$ is a vertical strip, and of a strictly decreasing word $w$. Such an object is weighted by $(-1)^{\left|\mu / \mu^{\prime}\right|}(-q)^{|w|}$.

Now apply Assaf-McNamara involution $\Phi$ to the tableau. More specifically, find $\Phi_{\lambda, \mu, \lambda^{\prime}, \mu^{\prime}}(S)$. This is well defined because $\lambda^{\prime} / \lambda$ is a horizontal strip and $\mu / \mu^{\prime}$ is a vertical strip. The result is a semistandard Young tableaux $S^{\prime}$ of some shape $\lambda^{\prime \prime} / \mu^{\prime \prime}$ with the property that $\left|\mu / \mu^{\prime \prime}\right|=\left|\mu / \mu^{\prime}\right| \pm 1$ unless $S=S^{\prime}$ is a fixed point. This cancels more terms. The ones that remain correspond to fixed points of $\Phi_{\lambda, \mu, \lambda^{\prime}, \mu^{\prime}}$, together with a strictly decreasing word $w$ and the weight $(-q)^{|w|}$. Each such fixed point consists of a semistandard Young tableau $R$ of shape $\lambda / \mu$, together with a weakly increasing word $v$ and a strictly decreasing word $w$. Such an object is weighted by $(-q)^{|w|}$. Furthermore, every such triple $(R, v, w)$ appears as a non-canceling term on the right. Indeed, insert the elements of $v$ into $R$ to get a semistandard Young tableau $S$ of shape $\lambda^{\prime} / \mu$ for some partition $\lambda^{\prime}$ so that $\lambda^{\prime} / \lambda$ is a horizontal strip; then insert the elements of $w$ into $S$ and color the new cells gray to get a colored tableau $T$ of shape $\lambda^{+} / \mu$ for some partition $\lambda^{+}$so that $\lambda^{+} / \lambda^{\prime}$ is a vertical strip. Then applying $\Psi$ and $\Phi$ to $T$ yields $(R, v, w)$.

It remains to enumerate all triples $(R, v, w)$. If we want $(v, w)$ to contain, say, $\tau_{i}$ copies of $i, 1 \leq$ $i \leq \ell$, we can choose any $j$-subset of $\{1, \ldots, \ell\}$ and put the elements in decreasing order to form $w$, and then put the remaining elements of the multiset $\left\{1^{\tau_{1}}, 2^{\tau_{2}}, \ldots, \ell^{\tau_{\ell}}\right\}$ in weakly increasing order to form $v$. Furthermore, the weight of $(R, v, w)$ for these $v$ and $w$ is $(-q)^{j}$. That means that the right-hand side of SQMNR" becomes, after cancellations, $s_{\lambda / \mu} \cdot\left(\sum_{\tau \vdash r}(1-q)^{\ell(\tau)} m_{\tau}\right)$, which is the left-hand side of SQMNR". 


\section{A proof via skew Littlewood-Richardson rule}

It is informative to use Lam-Lauve-Sotille's [5] skew Littlewood-Richardson rule to find another proof of SQMNR. We need three lemmas. The first lemma is a simple computation that allows us to replace the quantum power sum functions with "hook" Schur functions. The second lemma is technical and states that a certain property is preserved in jeu de taquin slides. And the third lemma sheds some light on connections between jeu de taquin, hooks, and decompositions of broken ribbons into vertical and horizontal strips.

Lemma 8 For all $r$, we have

$$
\widetilde{p}_{r}=\sum_{k=1}^{r}(-q)^{r-k} s_{k, 1^{r-k}} .
$$

For the second lemma, we have to recall the celebrated backward (respectively, forward) jeu de taquin slide due to Schützenberger. Say we are given a standard Young tableau of shape $\lambda / \mu$. Let $c=c_{0}$ be a cell that is not in $\lambda / \mu$, shares the right or lower edge (respectively, the left or upper edge) with $\lambda / \mu$, and such that $\lambda / \mu \cup c$ is a valid skew diagram. Let $c_{1}$ be the cell of $\lambda / \mu$ that shares an edge with $c_{0}$; if there are two such cells, take the one with the smaller entry (respectively, larger entry). Then move the entry occupying $c_{1}$ to $c_{0}$, look at the tableau entries below or to the right of $c_{1}$ (respectively, above or to the left of $c_{1}$ ), and repeat the same procedure. We continue until we reach the boundary of $\lambda / \mu$, say in $m$ moves. The new tableau is a standard Young tableau and is called jdt ${ }_{c}(T)$. We say that $c_{0}, c_{1}, \ldots, c_{m}$ is the path of the slide.

If $T$ is a standard Young tableau of skew shape, we can repeatedly perform backward jeu de taquin slides. The final result $S$ is a standard Young tableau of straight shape, and it is independent of the choices during the execution of the algorithm. We say that $T$ rectifies to $S$. See [7, Appendix A1.2] for details and examples.

We say that a standard Young tableau $T$ of shape $\lambda / \mu$ has the $k$-NE property if the following hold:

NE1 the entry in the last cell of the first non-empty row (i.e. the northeast cell) of $\lambda / \mu$ is $k$;

NE2 if $i<j<k$, then $i$ appears strictly to the left of $j$ in $T$;

NE3 if $j>i>k$, then $i$ appears strictly above $j$ in $T$.

Lemma 9 If a tableau $T$ has the $k$-NE property, its shape is a broken ribbon. Furthermore, the $k$-NE property is preserved in a jeu de taquin slide.

Lemma 10 Take $r, k, 1 \leq k \leq r$, and let $S$ be the standard Young tableau of shape $\left(k, 1^{r-k}\right)$ with $1,2, \ldots, k$ in the first row, and $k+1, k+2, \ldots, r$ in rows $2,3, \ldots, r-k+1$. Choose a skew shape $\lambda / \mu$. Then the number of standard Young tableaux of shape $\lambda / \mu$ that rectify to $S$ is $\left(\begin{array}{c}\operatorname{rib}(\lambda / \mu)-1 \\ k-1-\operatorname{wt}(\lambda / \mu)\end{array}\right)$ if $\lambda / \mu$ is a broken ribbon of size $r$, and 0 otherwise.

Sketch of proof: Let us assume that $\lambda / \mu$ is a broken ribbon of size $r$ and count the number of standard Young tableaux of shape $\lambda / \mu$ that have the $k$-NE property. Place $k$ in the northeast cell. If a cell in $\lambda / \mu$ has a right neighbor in $\lambda / \mu$, then the entry has to be less than $k$ (otherwise both this entry and the entry to the right would be greater than $k$, and this would contradict NE3). Similarly, if a cell in $\lambda / \mu$ has an upper neighbor in $\lambda / \mu$, then the entry has to be greater than $k$ (otherwise both this entry and the entry above it 
would be less than $k$, and this would contradict NE2).

This means that there are at least $\operatorname{wt}(\lambda / \mu)$ elements that are $<k$. We can choose the northeast element of any ribbon except the northeast ribbon and make it $<k$. Since there are $k-1$ elements total that are less than $k$, we have $\left(\begin{array}{c}\operatorname{rib}(\lambda / \mu)-1 \\ k-1-\operatorname{wt}(\lambda / \mu)\end{array}\right)$ choices. The rest of the proof is easy and is left as an exercise for the reader.

Finally, recall the following result from [5]. For standard Young tableaux $T$ and $S$, we let $T * S$ be the tableau we get by placing $T$ below and to the left of $S$.

Theorem 11 (Skew Littlewood-Richardson rule - SLRR) Let $\lambda, \mu, \sigma, \tau$ be partitions and fix a tableau $S$ of shape $\sigma$. Then

$$
s_{\lambda / \mu} s_{\sigma / \tau}=\sum(-1)^{\left|R^{-}\right|} s_{\lambda^{+} / \mu^{-}}
$$

where the sum is over triples $\left(R^{-}, R^{+}, R\right)$ of standard Young tableaux of respective shapes $\left(\mu / \mu^{-}\right)^{c}$, $\lambda^{+} / \lambda$ and $\tau$ such that $R^{-} * R^{+} * R$ rectifies to $S$.

The lemmas indeed prove SQMNR as follows. By Lemma 8 and SLRR,

$$
s_{\lambda / \mu} \cdot \widetilde{p}_{r}=\sum_{k=1}^{r}(-q)^{r-k} s_{\lambda / \mu} \cdot s_{k, 1^{r-k}} . \quad s_{\lambda / \mu} \cdot s_{k, 1^{r-k}}=\sum_{R^{-}, R^{+}}(-1)^{\left|R^{-}\right|} s_{\lambda^{+} / \mu^{-}},
$$

where the last sum is over $R^{-} \in \mathrm{SYT}\left(\left(\mu / \mu^{-}\right)^{c}\right), R^{+} \in \mathrm{SYT}\left(\lambda^{+} / \lambda\right)$ such that $R^{-} * R^{+}$rectifies to $S$, where $S$ is the standard Young tableau of shape $\left(k, 1^{r-k}\right)$ with $1,2, \ldots, k$ in the first row, and $k+1, k+2, \ldots, r$ in rows $2,3, \ldots, r-k+1$. By Lemma 10 the sum on the right is over $\lambda^{+}, \mu^{-}$such that $\lambda^{+} / \lambda$ and $\mu / \mu^{-}$are broken ribbons, and for such $\lambda^{+}, \mu^{-}$, the coefficient of $s_{\lambda^{+} / \mu^{-}}$ is $(-1)^{\left|\mu / \mu^{-}\right|}\left(\begin{array}{c}\operatorname{rib}\left(\lambda^{+} / \lambda\right)+\operatorname{rib}\left(\mu / \mu^{-}\right)-1 \\ k-1-\operatorname{wt}\left(\lambda^{+} / \lambda\right)-\operatorname{ht}\left(\mu / \mu^{-}\right)\end{array}\right)$. This means that the coefficient of $s_{\lambda^{+} / \mu^{-}}$in $s_{\lambda / \mu} \cdot \widetilde{p}_{r}$ is

$$
(-1)^{\left|\mu / \mu^{-}\right|} \sum_{k}(-q)^{r-k}\left(\begin{array}{c}
\operatorname{rib}\left(\lambda^{+} / \lambda\right)+\operatorname{rib}\left(\mu / \mu^{-}\right)-1 \\
k-1-\operatorname{wt}\left(\lambda^{+} / \lambda\right)-\operatorname{ht}\left(\mu / \mu^{-}\right)
\end{array}\right) .
$$

Since $r=\operatorname{rib}\left(\lambda^{+} / \lambda\right)+\operatorname{rib}\left(\mu / \mu^{-}\right)+\operatorname{wt}\left(\lambda^{+} / \lambda\right)+\operatorname{wt}\left(\mu / \mu^{-}\right)+\operatorname{ht}\left(\lambda^{+} / \lambda\right)+\operatorname{ht}\left(\mu / \mu^{-}\right)$, the sum equals

$$
\begin{gathered}
(-q)^{\mathrm{ht}\left(\lambda^{+} / \lambda\right)+\operatorname{wt}\left(\mu / \mu^{-}\right)} \sum_{k}(-q)^{\operatorname{rib}\left(\lambda^{+} / \lambda\right)+\operatorname{rib}\left(\mu / \mu^{-}\right)-1-\left(k-1-\operatorname{wt}\left(\lambda^{+} / \lambda\right)-\operatorname{ht}\left(\mu / \mu^{-}\right)\right)}\left(\begin{array}{c}
\operatorname{rib}\left(\lambda^{+} / \lambda\right)+\operatorname{rib}\left(\mu / \mu^{-}\right)-1 \\
k-1-\operatorname{wt}\left(\lambda^{+} / \lambda\right)-\operatorname{ht}\left(\mu / \mu^{-}\right)
\end{array}\right) \\
=(-q)^{\operatorname{ht}\left(\lambda^{+} / \lambda\right)+\operatorname{wt}\left(\mu / \mu^{-}\right)}(1-q)^{\operatorname{rib}\left(\lambda^{+} / \lambda\right)+\operatorname{rib}\left(\mu / \mu^{-}\right)-1}
\end{gathered}
$$

by the binomial theorem. This is SQMNR'.

\section{Final remarks}

The motivation for this work was the open problem posed by Assaf and McNamara in [2]: to find a combinatorial proof of the skew Murnaghan-Nakayama rule. Even though this paper provides a completely bijective proof of the skew quantum Murnaghan-Nakayama rule, which obviously specializes to the nonquantum rule, Assaf-McNamara's problem remains open. Indeed, plugging $q=1$ into SQMNR", which 
is the identity we proved bijectively, gives 0 on both sides. To get SMNR, we have to divide SQMNR" by $1-q$ and then set $q=1$.

A more algebraic (and shorter) proof of SQMNR would run as follows. There is a short algebraic proof of SPR due to Lam in the appendix of [1]. We have already mentioned that CSPR follows by applying the involution $\omega$ to SPR. It is easy to prove that $\sum_{\tau \vdash r}(1-q)^{\ell(\tau)} m_{\tau}=\sum_{k=0}^{r}(-q)^{r-k} s_{k} s_{1^{r-k}}$. So by SPR

and CSPR, we have $s_{\lambda / \mu} \cdot\left(\sum_{\tau \vdash r}(1-q)^{\ell(\tau)} m_{\tau}\right)=\sum(-1)^{\left|\mu / \mu^{-}\right|}(-q)^{\left|\lambda^{+} / \lambda^{\prime}\right|+\left|\mu^{\prime} / \mu^{-}\right|} s_{\lambda^{+} / \mu^{-}}$, where the sum is over partitions $\lambda^{+}, \lambda^{\prime}, \mu^{-}, \mu^{\prime}$ such that $\lambda^{\prime} / \lambda$ and $\mu^{\prime} / \mu^{-}$are horizontal strips, $\lambda^{+} / \lambda^{\prime}$ and $\mu / \mu^{\prime}$ are vertical strips, $\left|\lambda^{+} / \lambda^{\prime}\right|+\left|\mu^{\prime} / \mu^{-}\right|=r-k$ and $\left|\lambda^{\prime} / \lambda\right|+\left|\mu / \mu^{\prime}\right|=k$. This implies SQMNR" and hence SQMNR via Lemma 7, which was proved independently of everything else in Section 3 .

Lam-Lauve-Sotille's skew Littlewood-Richardson rule is very general, but the computation of actual coefficients in the expansion, i.e. counting all standard Young tableaux of a given shape that rectify to a given tableau, is complicated in practice. In light of Section 4, our work can be seen as one possible answer to the following question. For what special shapes of $\lambda, \mu, \sigma, \tau$ can we actually compute the coefficients? SQMNR can be interpreted as saying that if $\tau=\emptyset$ and $\sigma$ is a hook, the coefficients are certain binomial coefficients, while SPR says that the coefficient is \pm 1 if $\tau=\emptyset$ and $\sigma=(r)$. It would be interesting to find other examples when the coefficients can be computed and yield elegant answers, both for Schur functions and for other Hopf algebras.

The quantum power sum functions $\widetilde{p}_{r}$ are special cases of the Hall-Littlewood polynomials. See [4, §5] for some conjectures involving Pieri rule-type products of (skew) Hall-Littlewood polynomials and Schur functions.

\section{Acknowledgments}

Peter McNamara's excellent talk [2] at FPSAC'10 was the main motivation for this work. Sage [6] was of great help in guessing the right form for SQMNR.

\section{References}

[1] S. Assaf and P. McNamara (with an appendix by T. Lam), A Pieri Rule for Skew Shapes, to appear in J. Combin. Theory, Ser. A, arXiv:0908.0345

[2] S. Assaf and P. McNamara, A Pieri Rule for Skew Shapes, slides from a talk at FPSAC 2010, available at http://linux.bucknell.edu/ pm040/Slides/McNamara.pdf

[3] M. Konvalinka, Combinatorics of determinental identities, Ph.D. thesis, MIT, Cambridge, Massachusetts, 2008, $129 \mathrm{pp}$.

[4] M. Konvalinka, Skew quantum Murnaghan-Nakayama rule, preprint (2010)

[5] T.Lam, A. Lauve and F. Sottile, Skew Littlewood-Richardson Rules from Hopf Algebras, Int. Math. Res. Notices, doi: 10.1093/imrn/rnq104 (2010)

[6] The Sage-Combinat community, Sage-Combinat: enhancing Sage as a toolbox for computer exploration in algebraic combinatorics, http: / / comb inat. sagemath.org, 2008.

[7] R. P. Stanley, Enumerative combinatorics, Vol. 2, Cambridge University Press, Cambridge, 1999 